\title{
Research on I / Q Unbalanced Channel Reciprocity Calibration Algorithm in Massive MIMO System
}

\author{
Jinming Cui ${ }^{1}$, Liang $\mathrm{Yin}^{1,}{ }^{*}$, Yue $\mathrm{Ma}^{2}$, Shufang $\mathrm{Li}^{1}$ \\ ${ }^{1}$ Beijing Key Laboratory of Network System Architecture and Convergence, Beijing University of Posts and Telecommunications, \\ 100876, Beijing, China \\ ${ }^{2}$ School of Computer Science, Beijing University of Posts and Telecommunications, 100876, Beijing, China
}

\begin{abstract}
Massive MIMO system uses the transmitter channel state information (CSI) to preprocess the transmitted data, which can effectively reduce the interference and enhance the Massive MIMO system capacity. In the actual transmission process, reciprocity is subject to many factors, resulting in application difficulties. In this paper, we mainly study the influence of RF device's noncardiosis on channel reciprocity of Massive MIMO system. Combined with channel estimation based on pilot signal and FRRH subspace tracking algorithm, this paper proposes a channel reciprocity calibration method for Massive MIMO. Finally, the compensation effect of the compensation algorithm is verified by simulation.
\end{abstract}

\section{Introduction}

Massive MIMO system uses the transmitter channel state information (CSI) to pre-process the transmitted data, which can effectively reduce the interference and enhance the Massive MIMO system capacity [1]. In the TDD system, the uplink and downlink data transmission uses the same frequency, and the uplink and downlink wireless channel environments are the same. Therefore, the base station can get the downlink channel CSI from the uplink channel CSI obtained by the uplink channel estimation, that is, the uplink and downlink channels are reciprocal $[2,3]$.

In the actual transmission process, reciprocity is subject to many factors, resulting in application difficulties. Channel estimation error, channel time-varying, nonideality of radio frequency devices and asymmetry of uplink and downlink interference modes will lead to a certain degree of loss of channel reciprocity, resulting in that the base station can not directly obatain the downlink channel CSI from uplink channel CSI $[4,5]$. In the case where the channel reciprocity is affected, it is necessary to use the channel estimation to compensate for the lack of reciprocity of the channel [6]. This paper mainly studies the influence of the non-ideality of RF devices on the channel reciprocity of Massive MIMO systems.

I / Q imbalance is caused by the non-ideal characteristics of the wireless transceiver circuit, which is present in the process of upconversion and down-conversion. The result is, the amplitude gain of the I / Q branch of the signal is not equal or the phase is not orthogonal $[7,8]$. In the presence of the I/ Q imbalance, the channel will lose its reciprocity even if the radio channel itself remains unchanged during an uplink and downlink subframe interaction.

[9] proposed a calibration method based on mutual coupling of time-division duplex massive MIMO system. [10] considered the channel estimation error to simulate and analyze the effect on TDD multi-user massive MIMO system linear precoding caused by RF mismatch. [11] analyzes the bidirectional signaling method of time division duplex reciprocal calibration (RC) in multi-user beamforming system. [12] and [13] proposed a new closed-loop reciprocal calibration method. Compared with the existing method, the proposed method can be more accurate to support online calibration.

In this paper, based on the above research, combined with the channel prediction, the impact of the RF devices' non-ideal channel reciprocity and compensation algorithm are simulated and researched.

\section{System model}

The system studied in this paper contains L cells, where each base station (BS) is configured with $\mathrm{M}$ antennas and has N single antenna users (UTs).

Foundation items: National Natural Science Foundation of China, China (No. 61427801)

* Corresponding author: yinl@bupt.edu.cn 


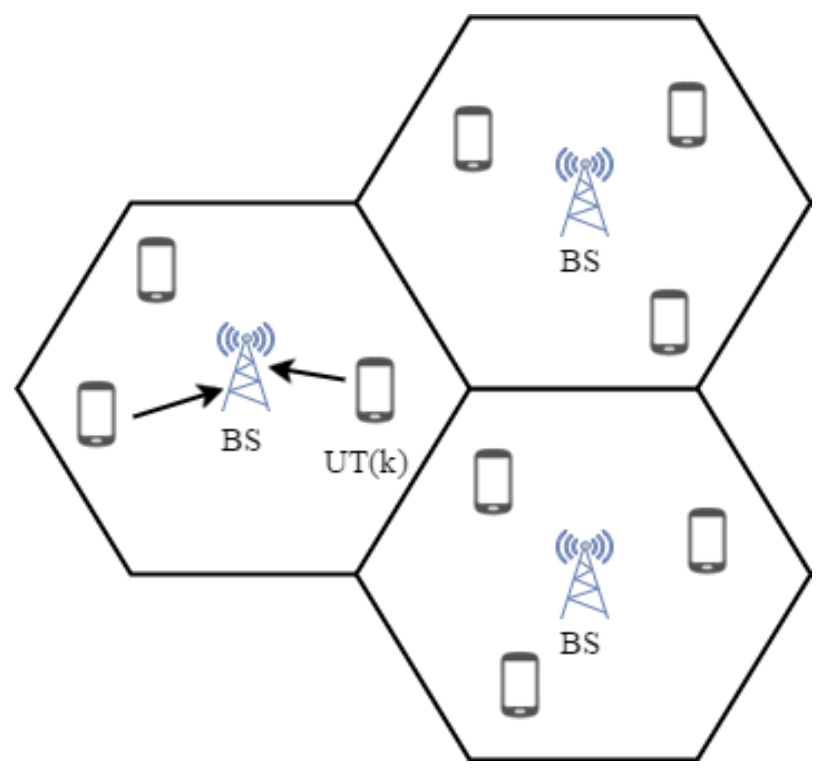

Fig. 1. System model diagram.

The SISO system model with I / Q unbalanced receiver is shown in Fig. 2.

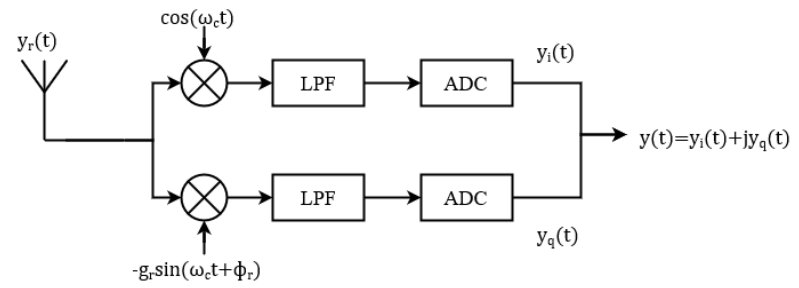

Fig. 2. I / Q unbalanced SISO system model.

The signal from the user to the base station (uplink baseband signal) can be expressed as:

$$
Y_{i}=K_{B i} H K_{U i} X+N_{\mathrm{W}}
$$

Where $\mathrm{i}=1, \ldots, \mathrm{L}$.

The transmit signal is:

$$
X=\left[\mathrm{x}_{1 \mathrm{I}}, \mathrm{x}_{1 \mathrm{Q}}, \cdots \mathrm{x}_{\mathrm{NI}}, \mathrm{x}_{\mathrm{NQ}}\right]
$$

Wherein the elements represent the transmission signals from the first to the N-th UT to the BS in the i-th cell.

$\mathrm{K}$ in the expression represents the I / Q unbalance matrix between the BS and the UT. The I / Q unbalance matrix at the BS side can be expressed as:

$$
K_{B i}=\left(\begin{array}{ccc}
K_{B i 1} & 0 & 0 \\
0 & \ddots & 0 \\
0 & 0 & K_{B i M}
\end{array}\right)
$$

Where the matrix elements are the I / Q unbalance parameters of the first to the M-th BS antennas at the BS in the i-th cell, respectively, and can be expressed as:

$$
K_{B i k}=\left(\begin{array}{cc}
\alpha_{B i k}\left(1+\beta_{B i k}\right) & -\alpha_{B i k} \sin \theta_{B i k} \\
0 & \alpha_{B i k} \cos \theta_{B i k}
\end{array}\right)
$$

Where $\mathrm{k}=1, \ldots, \mathrm{M}$.

The UT side I / Q unbalance matrix can be expressed as:

$$
K_{U i}=\left(\begin{array}{ccc}
K_{U i 1} & 0 & 0 \\
0 & \ddots & 0 \\
0 & 0 & K_{U i N}
\end{array}\right)
$$

Where the matrix elements are the I / Q unbalance parameters of the first to N-th UTs in the i-th cell, respectively, and can be expressed as:

$$
K_{U i l}=\left(\begin{array}{cc}
\alpha_{U i l}\left(1+\beta_{U i l}\right) & -\alpha_{U i l} \sin \theta_{U i l} \\
0 & \alpha_{U i l} \cos \theta_{U i l}
\end{array}\right)
$$

Where $1=1, \ldots, \mathrm{N}$

$\alpha$ in the I / Q unbalanced parameter is the power holding factor, $\beta$ is the difference ratio of I / Q signal, $\theta$ is the phase difference of I / Q signal.

In the expression, $\mathbf{H}$ is the channel environment between the BS and the UT, and can be expressed as:

$$
\mathrm{H}=\left(\begin{array}{ccccc}
\mathrm{h}_{11 \mathrm{I}} & -\mathrm{h}_{11 \mathrm{Q}} & \cdots & \mathrm{h}_{1 \mathrm{NI}} & -\mathrm{h}_{1 \mathrm{NQ}} \\
\mathrm{h}_{11 \mathrm{Q}} & \mathrm{h}_{11 \mathrm{I}} & \cdots & \mathrm{h}_{1 \mathrm{NQ}} & \mathrm{h}_{1 \mathrm{NI}} \\
\vdots & \vdots & \ddots & \vdots & \vdots \\
\mathrm{h}_{\mathrm{M} 1 \mathrm{I}} & -\mathrm{h}_{\mathrm{M} 1 \mathrm{Q}} & \cdots & \mathrm{h}_{\mathrm{MNI}} & -\mathrm{h}_{\mathrm{MNQ}} \\
\mathrm{h}_{\mathrm{M} 1 \mathrm{Q}} & \mathrm{h}_{\mathrm{M} 1 \mathrm{I}} & \cdots & \mathrm{h}_{\mathrm{MNQ}} & \mathrm{h}_{\mathrm{MNI}}
\end{array}\right)
$$

$\mathbf{N}_{\mathbf{w}}$ in the expression is noise, subject to the Gaussian distribution and is independent of the user channel.

\section{Compensation algorithm}

\subsection{Channel estimation and compensation}

Using pilot signals:

$$
\mathbf{Y}_{\mathbf{p}}(t)=\mathbf{H S} \mathbf{S}_{\mathbf{p}}+\mathbf{n}_{\mathbf{p}}(t)+\mathbf{n}_{\mathbf{p}}{ }^{*}(t)
$$

where:

$$
\begin{aligned}
& \mathbf{H}=\left(\mathbf{h}(\mathrm{t}) \quad \mathbf{h}^{*}(\mathrm{t})\right) \\
& \mathbf{S}_{\mathbf{p}}=\left(\mathbf{x}_{\mathbf{p}}(\mathrm{t}) \quad \mathbf{x}_{\mathbf{p}}^{*}(\mathrm{t})\right)^{T}
\end{aligned}
$$

The channel estimation result of the LS estimation is:

$$
\mathbf{H}=\mathbf{Y}_{\mathbf{p}}(t) \mathbf{S}_{\mathbf{p}}{ }^{H}\left(\mathbf{S}_{\mathbf{p}} \mathbf{S}_{\mathbf{p}}{ }^{H}\right)^{-1}
$$

The mean square error (MSE) is:

$$
\begin{aligned}
& \sigma_{\Omega}^{2}=E\left[\|\mathbf{H}-\mathbf{H}\|_{F}^{2}\right] \\
& =E\left[\left\|\left(\mathbf{n}_{\mathbf{p}}(t)+\mathbf{n}_{\mathbf{p}}{ }^{*}(t)\right) \mathbf{S}_{\mathbf{p}}{ }^{H}\left(\mathbf{S}_{\mathbf{p}} \mathbf{S}_{\mathbf{p}}{ }^{H}\right)^{-1}\right\|_{F}^{2}\right] \\
& =2 \mathrm{n}_{\mathrm{R}} \mathrm{N}_{0} \operatorname{tr}\left[\left(\mathbf{S}_{\mathbf{p}} \mathbf{S}_{\mathbf{p}}{ }^{H}\right)^{-1}\right]
\end{aligned}
$$

To minimize the MSE, the rows of the matrix SP should be orthogonal, and the equivalent channel estimates can be further obtained: 


$$
\mathbf{H}_{e q}=\left(\begin{array}{l}
y_{p} \\
\mathrm{y}_{\mathrm{p}}^{*}
\end{array}\right)\left(\left(\begin{array}{l}
x_{p} \\
\mathrm{x}_{\mathrm{p}}^{*}
\end{array}\right)^{H}\left(\left(\begin{array}{l}
x_{p} \\
\mathrm{x}_{\mathrm{p}}^{*}
\end{array}\right)\left(\begin{array}{l}
x_{p} \\
\mathrm{x}_{\mathrm{p}}^{*}
\end{array}\right)^{H}\right)^{-1}\right)
$$

\subsection{FRRH Channel estimation algorithm}

In the Massive MIMO system, due to the large number of antennas and the large scale, the traditional channel estimation method is not efficienct enough. Therefore, a low-complexity semi-blind channel estimation method based on subspace tracking algorithm is introduced: FRRH (Fast Recursive Row-Householder) channel estimation algorithm.

Using the FRRH algorithm, the fuzzy matrix is estimated as follows:

$$
\hat{\Theta}(n)=\left[\left(U^{S}(n)\right)^{\mathrm{H}} U^{S}(n)\right]^{-1}\left(U^{S}(n)\right)^{\mathrm{H}} \hat{H}_{e q}
$$

Where $U^{S}$ is the signal subspace of the receiver pilot signal.

The channel estimation expression is:

$$
\begin{aligned}
& \hat{H}(n)=U^{S}(n) \hat{\Theta}(n) \\
& =U^{S}(n)\left[\left(U^{S}(n)\right)^{\mathrm{H}} U^{S}(n)\right]^{-1}\left(U^{S}(n)\right)^{\mathrm{H}} \hat{H}_{e q}
\end{aligned}
$$

Through the recursive channel estimation, the computational efficiency can be greatly improved, and the accurate channel estimation result can be obtained, and the channel reciprocity calibration is carried out.

\section{Simulation analysis}

\subsection{Simulation environment}

It is assumed that the base station (BS) in each cell is configured with 64 antennas and has 8 single antenna users (UTs).

The antenna parameters are set as follows:

Table 1. $64 \times 8$ antenna parameter settings.

\begin{tabular}{|c|c|c|c|}
\hline$\beta_{\text {Bik }}$ & $\theta_{\text {Bik }}$ & $\beta_{\text {Uil }}$ & $\theta_{\text {Uil }}$ \\
\hline 1.15 & $5^{\circ}$ & 1.05 & $10^{\circ}$ \\
\hline
\end{tabular}

Note: $\mathrm{k}=1, \cdots, 64 ; 1=1, \cdots, 8$

It is assumed that the base station (BS) in each cell is configured with 128 antennas and has 16 single antenna users (UTs).

The antenna parameters are set as follows:

Table 2. $128 \times 16$ antenna parameter settings.

\begin{tabular}{|c|c|c|c|}
\hline$\beta_{\text {Bik }}$ & $\theta_{\text {Bik }}$ & $\beta_{\text {Uil }}$ & $\theta_{\text {Uil }}$ \\
\hline 1.15 & $5^{\circ}$ & 1.05 & $10^{\circ}$ \\
\hline
\end{tabular}

Note: $\mathrm{k}=1, \ldots, 128 ; 1=1, \ldots, 16$
In this paper, the bit error rate and channel capacity are used as the parameters to measure the effect and compensation effect. In this paper, the water capacity algorithm is used to calculate the channel capacity:

$$
\mathrm{C}=\frac{1}{2} E\left\{\sum_{i=1}^{M} \log _{2}\left(1+\frac{P_{S i}\left|\beta_{i 1}\right|^{2}}{\mathrm{P}_{N}+\sum_{j=1, j \neq i}^{M} P_{S j}\left|\beta_{i j}\right|^{2}}\right)\right\}
$$

\subsection{Simulation results}

The channel capacity comparison result of $64 \times 8$ antenna-scale Massive MIMO system's ideal I / Q channel case and imbalanced I / Q channel case is shown in Figure 3:

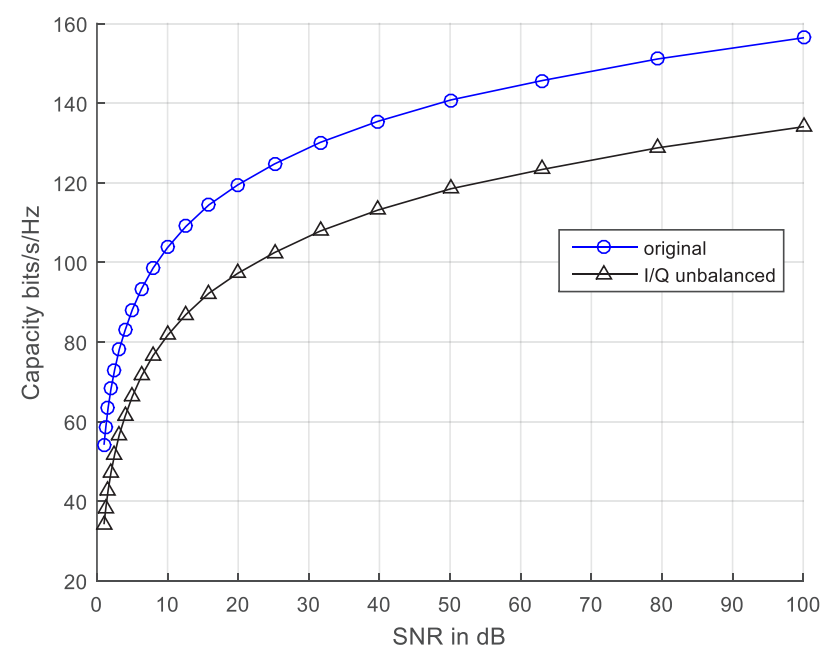

Fig. 3. Channel capacity comparison result.

Using the channel analysis of the unbalanced I / Q channel compensation method, the channel reciprocity can be compensated. Figure 4 shows the channel capacity before and after the compensation:

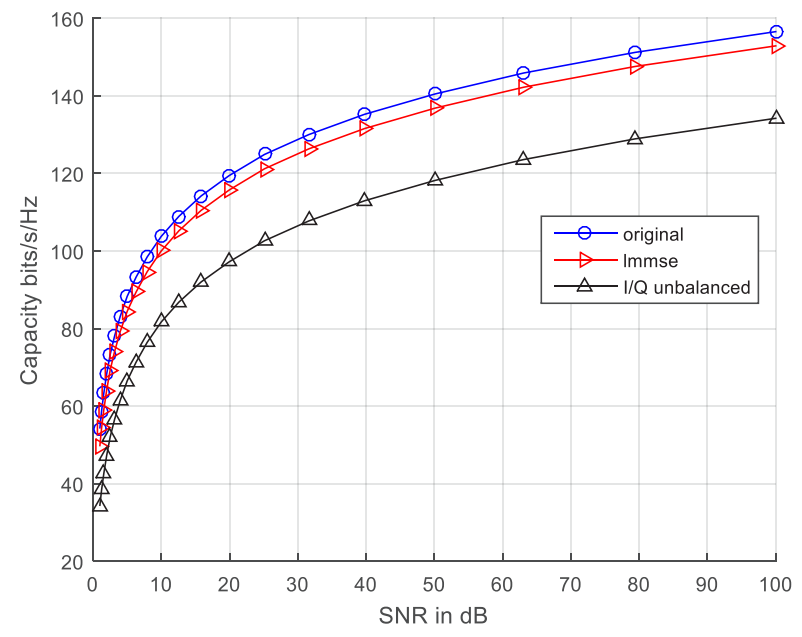

Fig. 4. Channel capacity before and after compensation.

Figure 5 shows the error rate before and after the compensation: 


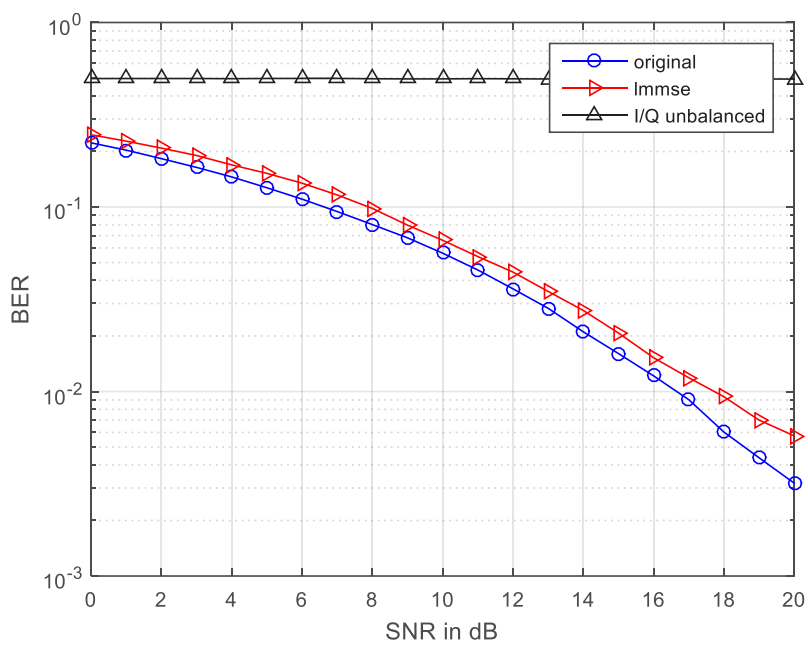

Fig. 5. Error rate before and after the compensation.

For the Massive MIMO system with an antenna scale of $128 \times 16$, Figure 6 shows the comparison of the channel capacity before and after compensation:

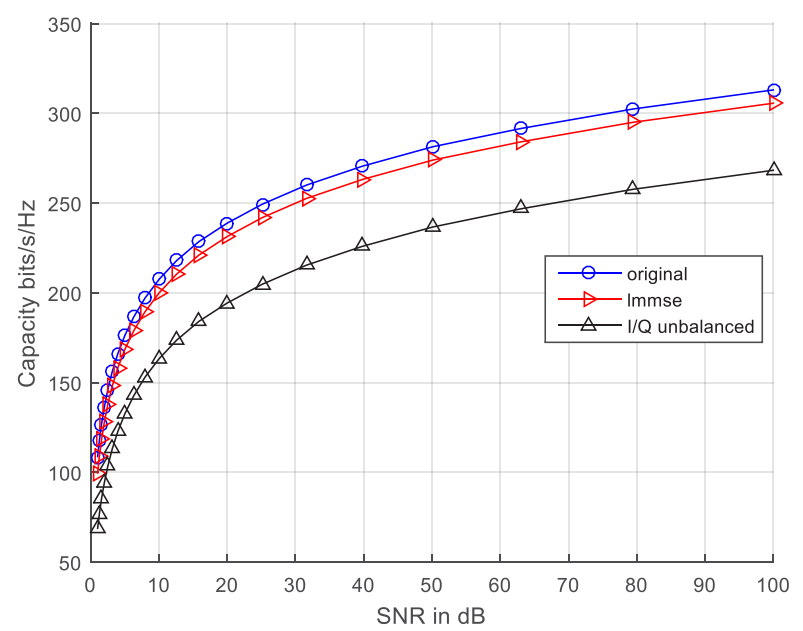

Fig. 6. Channel capacity comparison result $(128 \times 16$ scale $)$.

Figure 7 shows the error rate before and after the compensation:

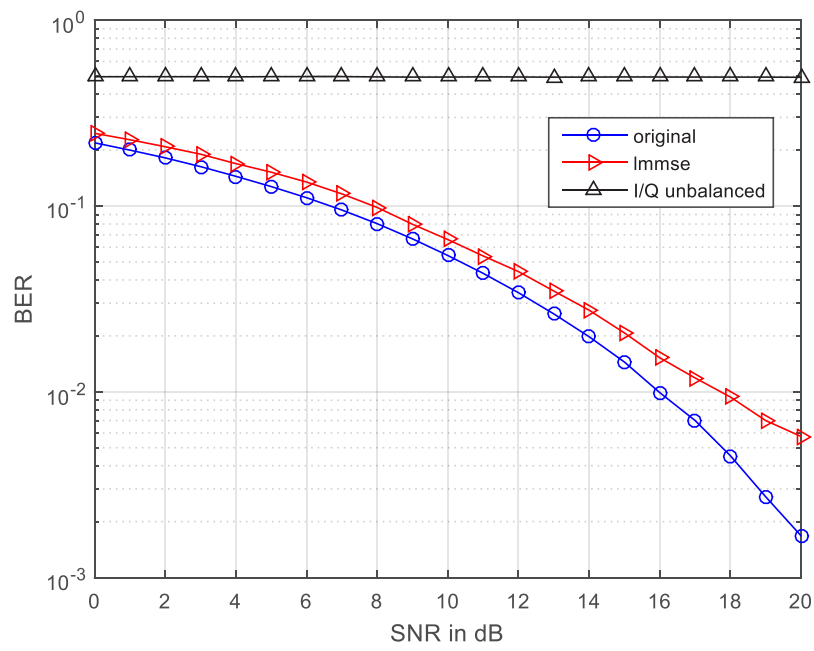

Fig. 7. Error rate before and after the compensation $(128 \times 16$
From the simulation analysis, it can be seen that the nonideality of the RF devices in the Massive MIMO system with $64 \times 8$ antenna scale has a great influence on the channel reciprocity both in terms of channel capacity and bit error rate. With the compensation algorithm proposed, the channel capacity and bit error rate can be nearly restored to the ideal RF device situation, to achieve a good compensation effect. While, for the Massive MIMO system with an antenna scale of $128 \times 16$, the compensation algorithm proposed in this paper can also achieve great compensation effect.

\section{Conclusions}

Based on the research of Massive MIMO channel estimation, the paper researches on the channel reciprocity of TDD Massive MIMO system, mainly on the effect of non-ideal RF device and the compensation method. The simulation results show that the compensation method proposed in this paper has great compensation effect on the channel reciprocity of Massive MIMO system. The further studies could be the verification of the compensation method in the semiphysical simulation platform, and lower complexity of the algorithm to reduce frequency pollution effects.

\section{References}

1. Marzetta T L. Noncooperative cellular wireless with unlimited numbers of base station antennas[J]. IEEE Transactions on Wireless Communications, 2010, 9(11):3590-3600.

2. $\mathrm{Fu}$ Xiaowei. Channel estimation and pilot scheduling of Massive MIMO systems under pilot pollution $[\mathrm{J}]$; Journal of Southwest Jiaotong University;

3. Liu Yijing. TDD system channel reciprocity analysis and application of [D]. Beijing University of Posts and Telecommunications, 2014.

4. He Fuli. TDD MIMO channel non-reciprocal compensation algorithm [D]. Xidian University, 2011.

5. Gao Q, Qin F, Sun S. Utilization of channel reciprocity in advanced MIMO system[C]// Communications and Networking in China (CHINACOM), 2010 5th International ICST Conference on. IEEE, 2010:1 - 5.

6. Bourdoux A, Come B, Khaled N. Nonreciprocal transceivers in OFDM/SDMA systems: impact and mitigation[C]// Radio and Wireless Conference, 2003. RAWCON '03. Proceedings. IEEE, 2003:183-186.

7. Björnson E, Matthaiou M, Debbah M. Massive MIMO with Non-Ideal Arbitrary Arrays: Hardware Scaling Laws and Circuit-Aware Design[J]. IEEE Transactions on Wireless Communications, 2014, 14(8):4353-4368.

scale). 
8. Zarei S, Gerstacker W H, Aulin J, et al. I / Q Imbalance Aware Widely-Linear Receiver for Uplink Multi-Cell Massive MIMO Systems: Design and Sum Rate Analysis[J]. IEEE Transactions on Wireless Communications, 2016, 15(5):3393-3408.

9. Vieira J, Rusek F, Edfors O, et al. Reciprocity Calibration for Massive MIMO: Proposal, Modeling and Validation[J]. 2016.

10. Mi D, Dianati M, Zhang L, et al. Massive MIMO Performance with Imperfect Channel Reciprocity and Channel Estimation Error[J]. IEEE Transactions on Communications, PP(99):1-1.

11. Liu D, Ma W, Shao S, et al. Performance Analysis of TDD Reciprocity Calibration for Massive MU-MIMO Systems With ZF Beamforming $[\mathrm{J}]$. IEEE Communications Letters, 2016, 20(1):113-116.

12. Luo H, Zhang Y, Huang L K, et al. A new reciprocity calibration method for massive MIMO systems[C]// IEEE International Symposium on Broadband Multimedia Systems and Broadcasting. IEEE, 2016:1-4.

13. Luo H, Zhang Y, Huang L K, et al. A ClosedLoop Reciprocity Calibration Method for Massive MIMO in Terrestrial Broadcasting Systems[J]. IEEE Transactions on Broadcasting, 2016, PP(99):1-9. 